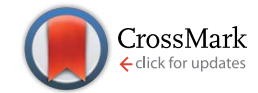

Cite this: RSC Adv., 2016, 6, 80773

\title{
Size-controlled synthesis of gold nanoparticles by a novel atmospheric pressure glow discharge system with a metallic pin electrode and a flowing liquid electrode
}

\begin{abstract}
A. Dzimitrowicz, ${ }^{a}$ K. Greda, ${ }^{a}$ T. Lesniewicz, ${ }^{b}$ P. Jamroz, ${ }^{a}$ M. Nyk ${ }^{c}$ and P. Pohl ${ }^{* a}$
A direct current atmospheric pressure glow discharge (APGD) operated between a pin-type solid metallic electrode and the surface of a flowing solution (the liquid electrode), positively or negatively charged and serving as the flowing liquid anode (FLA) or cathode (FLC), was used for synthesizing gold nanoparticles (AuNPs). To the best of our knowledge, the synthesis of AuNPs in such a system, with no noble gas required to support the APGD, has never been reported. The effect of the selected operating parameters on the performance of the AuNPs synthesis in the plasma-reaction system with the FLA or FLC was examined. The design of the experiments (DOE) was conducted using the response surface regression (RSR) approach. The response of both systems was the wavelength of the maximum $\left(\lambda_{\max }\right)$ of the localized surface plasmon resonance (LSPR) absorption band of the AuNPs. On the basis of the established full quadratic regression models, the optimal operating parameters for both plasma-reaction systems (with the FLA or the FLC) were selected, which allowed for the smallest in size spherical AuNPs to be obtained. Both regression models were validated, the AuNPs produced in both plasma-reaction systems under the optimal operating parameters were characterized by UV-Vis absorption spectrophotometry, scanning electron microscopy (SEM) and transmission electron microscopy (TEM).
\end{abstract}

Received 11th July 2016

Accepted 9th August 2016

DOI: $10.1039 / c 6 r a 17706 a$

www.rsc.org/advances

\section{Introduction}

Conventional methods for the synthesis of metallic nanoparticles (NPs) based on colloidal chemistry typically require many processing stages, including a cleaning step of the resulting nanofluids with various solvents. ${ }^{1}$ Unfortunately, multi-step methods are less feasible for industrial applications and this limits their throughput. ${ }^{2}$ Additionally, potentially toxic chemical reagents, e.g. $\mathrm{NaBH}_{4}$, are often used to reduce the NP precursors, making the synthesized metal nanocolloids unsafe and bioincompatible. ${ }^{\mathbf{1} 3}$ Finally, the necessity to separate the NPs and clean them from the excess of the reducing agents can result in the irreversible contamination of their surface with different impurities. ${ }^{2}$

In response to the above mentioned drawbacks of the traditional colloidal chemistry synthesis methods, the last

${ }^{a}$ Wroclaw University of Technology, Faculty of Chemistry, Department of Analytical Chemistry and Chemical Metallurgy, Wybrzeze Stanislawa Wyspianskiego 27, 50-370 Wroclaw, Poland. E-mail: pawel.pohl@pwr.edu.pl

${ }^{b}$ OpEx (Six Sigma) Master Black Belt Independent Consultant, Klodzka 1f/1, 55-040 Bielany Wroclawskie, Poland

${ }^{c}$ Wroclaw University of Technology, Faculty of Chemistry, Department of Advanced Materials Engineering and Modelling, Wybrzeze Stanislawa Wyspianskiego 27, 50370 Wroclaw, Poland decade of research activity in nanotechnology has brought a new alternative solution to metallic NP synthesis. It is a plasma-mediated, single-step, green synthesis process that takes place at the plasma-liquid interface of the atmospheric pressure glow discharge (APGD) generated between gaseous jets and the surfaces of the liquids containing the metallic precursors. $^{4,5}$

The advantages of the plasma-assisted metallic NPs synthesis method are its simplicity, efficiency, low operation and material costs and the rapidness of the metallic NP nucleation and further growth. ${ }^{1}$ Indeed, the APGDs generated in contact with the relevant liquids are typically sustained in opento-air atmosphere without any expensive vacuum equipment and can be operated with very low electric power. ${ }^{6,7}$ The discharge currents accompanying the normal operation of the APGDs are commonly within 1-5 mA., ${ }^{1,2,8-20}$ Higher discharge currents, e.g. $8,{ }^{21} 10,{ }^{22} 13,{ }^{23,24}$ and $15 \mathrm{~mA},{ }^{21}$ are also used. The sustaining voltages change from 290 to $450 \mathrm{~V},^{12,17,23,24}$ but in general they are not higher than 800-2000 V. ${ }^{\mathbf{1 , 2 , 8 , 9 , 1 3 - 1 5 , 2 2}}$ The rapid and single-step production of the metallic NPs in the solutions is possible under ambient conditions because highenergy electrons from the discharge reach the surface of the liquid and, depending on their kinetic energy, participate in forming the various species, e.g. $\mathrm{H}_{2} \mathrm{O}_{2}, \mathrm{OH}$, or they simply 
become solvated electrons. ${ }^{\mathbf{8} 25}$ Both the species and/or solvated electrons can reduce the metallic NP precursors dissolved in the solutions and, as a result, the metal nanocolloids are instantly formed.

The construction of the open-to-air plasma-reaction chamber is relatively uncomplicated and inexpensive. ${ }^{4,5}$ In a typical plasma-reaction setup, a stable APGD is initiated between a low-flow gaseous jet cathode and a counter electrode, which is the liquid with an anode immersed into it, i.e. a graphite rod, ${ }^{\mathbf{1 , 8 , 9 , 1 3 - 1 5 , 1 7}} \mathrm{Pt}$ foil,, ${ }^{\mathbf{2 , 1 0 - 1 2 , 1 6 , 2 1 , 2 3 , 2 4}}$ a stainless-steel (SS) rod, ${ }^{8}$ a SS disk, ${ }^{22}$ or a Ni wire. ${ }^{8}$ To sustain the gaseous jet, $\mathrm{SS}^{1,2,8-13,16-22} \mathrm{Cu},{ }^{6,23,24}$ or $\mathrm{Ni}^{14,15}$ capillary tubes are used and Ar, ${ }^{1,6,10-12,14,15,23,24} \mathrm{He}^{\mathbf{2 , 8 , 9 , 1 3 , 1 6 - 2 1}}$ or He mixed with $\mathrm{H}_{2}$ (ref. 22) are passed through the tubes at a relatively low flow rate, i.e. 10-50

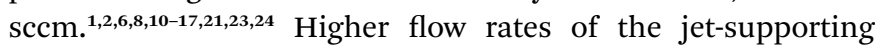
gases are also applied, i.e. $200 \mathrm{sccm}^{18-20}$ and $600 \mathrm{sccm}^{22}$ or even 1000-1100 $\mathrm{sccm}^{\mathbf{1 8 , 1 9}}$ when another jet sheath flow is used. The capillary tube is pointed downward, perpendicular to the surface of the liquid, grounded and negatively biased at a direct current high voltage (dc-HV). This makes the gaseous jet serve as the cathode of the discharge system and as the source of the high-energy electrons available for the plasma-liquid interactions. Instead of a solid anode, another gaseous jet can be applied. ${ }^{18-20}$ In this case, the metallic NPs are generated by irradiating the surface of the liquid not only with electrons but also with positive ions coming from the positively charged gaseous jet anode. ${ }^{18-20}$ The gap between the capillary tube tip and the liquid surface is usually 1-2 mm, ${ }^{1,2,6,10-12,14-16,18-21,23,24}$ but it can also be a bit lower (e.g. $0.3 \mathrm{~mm},{ }^{17} 0.7 \mathrm{~mm},{ }^{\mathbf{8 , 9 , 1 3}}$ and $0.9 \mathrm{~mm}$ (ref. 8)) or higher (5 mm (ref. 22)).

The APGD-based plasma-reaction systems and the plasmaliquid interactions accompanying them have thus far been used to synthesize AuNPs, ${ }^{6,8-11,13,18-21}$ AgNPs, ${ }^{10-12,16-20,24}$ alloyed $\mathrm{Au}_{x} \mathrm{Ag}_{1-x} \mathrm{NPs},{ }^{2}$ core-shell Ag@AuNPs, ${ }^{20}$ PtNPs, ${ }^{22}$ as well as $\mathrm{Fe}_{2} \mathrm{O}_{3} \mathrm{NPs},{ }^{18} \mathrm{Fe}_{3} \mathrm{O}_{4} \mathrm{NPs},{ }^{18,23}$ or $\mathrm{Fe}$ oxide nanoclusters. ${ }^{1}$ Fructose,, $\mathbf{2 , 1 0 , 1 1 , 1 6 , 2 1}$ sucrose, ${ }^{17}$ dextran, ${ }^{\mathbf{1 , 2 4}}$ citric acid, ${ }^{6}$ or sodium dodecyl sulfate ${ }^{18-20}$ are applied to prevent uncontrolled particle growth and the agglomeration of the synthesized metallic NPs. Additionally, the APGDs generated in contact with liquid media were also used to modify the surface properties of the Si nanocrystals (SiNCs). ${ }^{\mathbf{8 , 1 4 , 1 5}}$

All cited plasma-reaction systems are non-flow-through since the solutions are in small vessels ${ }^{1,2,8-17,21,22}$ or $\mathrm{U}^{\mathbf{6 , 2 3 , 2 4}}$ and $\mathrm{H}$-shaped ${ }^{18-20}$ cells, and the synthesis is commonly performed within 1 to $30 \mathrm{~min}$. To enhance this limited through-put of the APGD-assisted synthesis method and to increase its scope for the industrial applications, our research group developed a flow-through system and fully optimized it to obtain the spherical AuNPs of the smallest diameter. ${ }^{26,27}$ The system consisted of a flowing liquid cathode (FLC), i.e. a solution containing $\mathrm{HAuCl}_{4}$ and gelatin, which was continuously introduced to the system through a quartz tube at $3.0-5.0 \mathrm{~mL} \mathrm{~min}{ }^{-1}$ and negatively charged through a Pt wire, and a gaseous jet anode, operated in Ar that was introduced at $60-180 \mathrm{sccm}$ through a SS capillary tube. This plasma-reaction system continuously produced the AuNPs when the solutions of the AuNP precursors were introduced to it. The discharge was generated across a 5.0 mm gap between the surface of the flowing solution and the tip of the SS capillary tube. The discharge current was in the range of 15-45 mA. At the same time, another flow-through system was described by Ghosh et al. and used for the production of the AgNPs. ${ }^{28}$ In this system an Ar jet, formed in a SS capillary tube and negatively biased, was perpendicularly positioned to a liquid jet containing $\mathrm{AgNO}_{3}$ and poly(acrylic) acid and positively biased through a woven Pt wire. The APGD was sustained in a $1 \mathrm{~mm}$ gap between the surface of the liquid jet and the SS capillary tube.

Since Ar or He gaseous jets are used as the cathodes or/and the anodes of the APGD-based plasma-reaction systems, the cost-effectiveness of the metallic NP synthesis in the overwhelming majority of the non-flow-through systems and flowthrough systems ${ }^{\mathbf{2 6 - 2 8}}$ is considerably reduced. Additionally, in the case of the non-flow-through systems, the yield of the metal nanocolloidal products is quite limited. Hence, the present work aimed to further develop and improve the APGD-based plasma-reaction systems to synthesize the metallic NPs on the example of Au. Here, to simplify the system design and reduce the operational costs, the APGD was fully sustained and stably operated across the gap between a pin-type solid metallic electrode and the surface of a flowing solution (the liquid electrode) that was positively or negatively charged and served as the flowing liquid anode (FLA) or cathode (FLC), respectively. No noble gas was required to sustain and operate the discharge. To the best of our knowledge, the synthesis of the AuNPs in such system has never been reported. To study the effect of the selected operating parameters on the performance of the AuNP synthesis in both systems, i.e. with the FLA or the FLC depending on the polarization of the electrodes, the design of experiments (DOE) along with the response surface regression (RSR) approach was used. The response of both systems was the wavelength of the maximum $\left(\lambda_{\max }\right)$ of the localized surface plasmon resonance (LSPR) absorption band of the AuNPs. On the basis of the full quadratic regression models, involving the main effects and the interactions of the studied parameters, the optimal operating parameters for both plasma-reaction systems (with the FLA or the FLC), which resulted in the spherical AuNPs with the smallest particle size, were selected. Both established regression models were validated, and the AuNPs obtained using the optimal parameters were characterized by UV-Vis absorption spectrophotometry, scanning electron microscopy (SEM) and transmission electron microscopy (TEM).

\section{Experimental}

\section{Reagents and solutions}

Re-distilled water was used for all preparations. Chemicals were analytical grade or better and used as received. Chloroauric acid tetrahydrate $\left(\mathrm{HAuCl}_{4} \cdot 4 \mathrm{H}_{2} \mathrm{O}\right)$ was obtained from Avantor Performance Materials (Gliwice, Poland) and used to prepare a $1000 \mu \mathrm{g} \mathrm{mL}{ }^{-1} \mathrm{Au}$ stock solution. To synthesize the AuNPs in the APGD-based plasma-reaction system, the working solutions to support the FLA or the FLC were prepared by appropriately diluting the Au stock solution. The working solutions contained 10.0, 105 or $200 \mu \mathrm{g} \mathrm{mL} \mathrm{m}^{-1}$ of the $\mathrm{Au}(\mathrm{III})$ ions. Additionally, to 
prevent the uncontrolled growth of the synthesized AuNPs, their aggregation and sedimentation, gelatin from bovine and porcine bones from Sigma-Aldrich (Steinheim, Germany) were used. This was present in the working solutions at a final concentration of $0,0.25$ or $0.50 \%(\mathrm{~m} / \mathrm{v})$. In the latter case, the appropriate amounts of gelatin powder were dissolved at first in the $50 \mathrm{~mL}$ portions of water, and then, after gelatin swelling, the resulting aliquots were warmed to $\sim 36^{\circ} \mathrm{C}$ and allowed to cool to room temperature. Finally, the appropriate amounts of the $\mathrm{Au}$ stock solution were added, and the working solutions were diluted with water to the required volume.

\section{Plasma-reaction system and the AuNP synthesis}

A schematic diagram of the flow-through plasma-reaction system using the APGD to synthesize the AuNPs is given in Fig. 1. The APGD was sustained and stably operated in the opento-air atmosphere with a $5.0 \mathrm{~mm}$ gap between a sharpened tip of a tungsten $(\mathrm{W})$ rod (id $4.0 \mathrm{~mm}$ ) and the surface of the flowing solution that was positively (the FLA) or negatively (the FLC) biased, as shown in Fig. 1a and b, respectively. The working solutions were delivered to the plasma-reaction system at flow

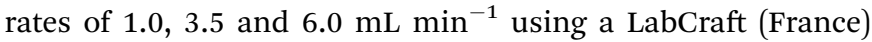
peristaltic pump, model Hydris 05. A quartz tube (id $2.0 \mathrm{~mm}$ ), onto which a graphite tube (id $4.0 \mathrm{~mm}$ ) was mounted, was used for this purpose. To charge the working solutions, a Pt wire was attached to the graphite tube. The overflowing solutions were collected in a small glass reservoir, which was a part of the liquid electrode casing, and were instantly drained out with the aid of another LabCraft Hydris 05 peristaltic pump. When the plasma-reaction system was working with the FLC, the solid pin electrode was positively biased at $900-1400 \mathrm{~V}$ (the anode of the system) using a dc-HV generator (Laboratory Electronic Instrumentation Production, Wroclaw, Poland). By changing the polarization of the electrodes, the plasma-reaction system functioned with the FLA, and the solid pin electrode acted as the cathode. Depending on the dc-HV used, the discharge current was changed within the range of 30 to $60 \mathrm{~mA}$. Additionally, the discharge current was stabilized by a $10 \mathrm{k} \Omega$ ballast resistor in the anode circuit.

The synthesis of the AuNPs in the plasma-reaction system operated with the FLA or the FLC was carried out in a continuous-flow mode by introducing the working solutions and sustaining the APGD. The overflowing, APGD-treated solutions, which contained the AuNPs produced at the given working conditions of the plasma-reaction system, were collected into PP stoppered tubes. The Au nanofluids were further characterized with respect to their optical properties (using UV-Vis absorption spectrophotometry) as well as their morphology and particle size (using SEM and TEM).

\section{Characterization of the synthesized AuNPs}

The optical properties of the AuNPs colloids were evaluated using UV-Vis absorption spectrophotometry. A double-beam UV-Vis Specord 210 Plus (Analytik Jena AG, Jena, Germany) instrument was applied. The spectral bandwidth of the spectrophotometer was set as $2 \mathrm{~nm}$ while the wavelength scan speed
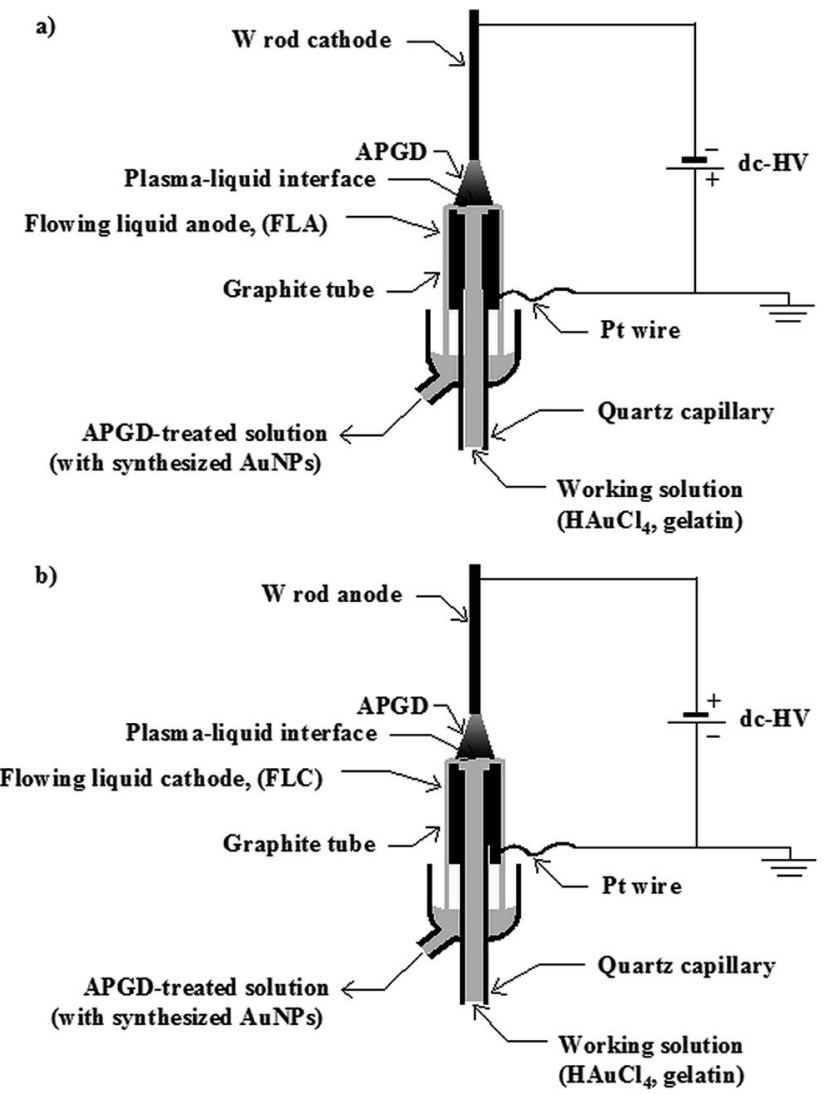

Fig. 1 The plasma-reaction systems based on the APGD sustained in the surrounding air between the solid pin electrode and the FLA (a) or the FLC (b).

was $12000 \mathrm{~nm} \mathrm{~min}^{-1}$. The measured absorption spectra gave information about the $\lambda_{\max }$ of the LSPR absorption band of the AuNPs, normally located within the range of $520-550 \mathrm{~nm}$ in the case of spherical AuNPs. ${ }^{29}$ According to the Mie scattering theory, ${ }^{28}$ the $\lambda_{\max }$ and its shift was used to elucidate changes in the particle size of the AuNPs synthesized under different working conditions of the plasma-reaction system. A blue shift in the $\lambda_{\max }$ indicated that the obtained AuNPs were of a smaller particle size. The absorption spectra of all the collected solutions containing the Au nanocolloids were acquired $24 \mathrm{~h}$ after the APGD treatment in the spectral range of $400-1100 \mathrm{~nm}$ versus re-distilled water as the blank. Additionally, the stability of the obtained AuNPs (stored at $4{ }^{\circ} \mathrm{C}$ in dark) was determined by measuring the position of the $\lambda_{\max }$ of the LSPR absorption band up to 4 months after the APGD treatment.

The first visual evidence of the morphology of the obtained AuNPs was attained using SEM. To provide good resolution for the SEM photomicrographs, the AuNPs present in the APGDtreated solutions were initially purified following a procedure recently described by Dzimitrowicz et al. ${ }^{26}$ A Joel (Joel USA Inc., Peabody, MA, USA) high-performance scanning electron microscope, model JSM-6610LVnx, was applied to characterize and image the AuNPs. The low vacuum pressure in the SEM chamber was within 10-270 Pa. The SEM micrographs were recorded using a magnification factor from 12000 to 33000 
when adjusting the accelerating voltage within the range from 20-28 kV. Two drops of the fluids containing the purified AuNPs were placed on C sticky tape and evaporated.

Due to the limited SEM resolution, TEM was used for determining the shape and the size as well as the size distribution of the obtained AuNPs. A FEI Tecnai G220 X-TWIN instrument (FEI, Hillsboro, OR, USA) equipped with an FEI energy dispersive X-ray microanalyzer (EDXMA) was applied. The samples for the TEM and EDXMA measurements were prepared by putting a drop of the fluids containing the purified AuNPs onto a GF Microsystems (Poznan, Poland) ultra-thin $\mathrm{Cu}$ substrate grid (CF400-Cu-UL, 400 mesh) and allowing it to dry under an IR lamp. To calculate the average particle size of the AuNPs, the ImageJ software (version 1.46 r, NIH) was applied.

\section{Optimization of the AuNP production by the design of experiments and response surface regression}

A Box-Behnken experimental design (BBD) was used to develop the second-order response surface model for the $\lambda_{\max }$, which was taken on the basis of the UV-Vis absorption spectra of the APGD-treated solutions containing the AuNPs, and to identify the optimum levels of the independent parameters that provided the lowest $\lambda_{\max }$ value referring to the spherical AuNPs of the smallest particle size. The BBD was selected in this particular case because it required fewer treatments than a central composite design (CCD) in the case of the system with 4 parameters. The mentioned continuously changed experimental parameters (factors), related to the performance of the plasma-reaction system and the course of the plasmaliquid interactions, were the flow rate of the flowing liquid

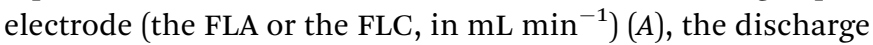
current (in $\mathrm{mA}$ ) $(B)$, the $\mathrm{Au}(\mathrm{III})$ ions concentration in the solution of the FLA or the FLC (in $\mu \mathrm{g} \mathrm{mL}{ }^{-1}$ ) $(C)$ and the gelatin concentration in this solution (in $\%, \mathrm{~m} / \mathrm{v}$ ) $(D)$. The levels of the factors $A, B, C$ and $D$ were fixed on the basis of the preliminary experimental trials and considering the stable operation of the APGD.

For modeling the response surface, the BBD was composed of 27 randomized treatments (runs) at 3 different levels $(+1,0$, -1 in coded units) and included 3 center points $(0,0,0)$. All the treatments were carried out in one block. The details of the runs with the input parameters are given in Table 1.

Table 1 The Box-Behnken design (BBD) with actual and coded values of the parameters ( $A$ - the flow rate of the flowing liquid electrode, $B$ the discharge current, $C$ - the $\mathrm{Au}(\mathrm{III})$ ion concentrations, $D$ - the gelatin concentration), the standardized and the randomized run orders and the response, i.e. the $\lambda_{\max }$ of the localized surface plasmon resonance (LSPR) absorption band of the AuNPs, of the plasma-reaction system working with the flowing liquid anode (FLA) or the cathode (FLC) ${ }^{a}$

\begin{tabular}{|c|c|c|c|c|c|c|c|c|c|}
\hline \multicolumn{2}{|l|}{ Run order } & \multicolumn{4}{|c|}{ Actual and (coded) levels of parameters } & \multicolumn{4}{|c|}{ Response $\left(\lambda_{\max }\right), \mathrm{nm}$} \\
\hline \multirow[b]{2}{*}{ Standardized } & \multirow[b]{2}{*}{ Randomized } & \multirow[b]{2}{*}{$A, \mathrm{~mL} \mathrm{~min}^{-1}$} & \multirow[b]{2}{*}{$B, \mathrm{~mA}$} & \multirow[b]{2}{*}{$C, \mu \mathrm{g} \mathrm{mL}^{-1}$} & \multirow[b]{2}{*}{$D, \%$} & \multicolumn{2}{|l|}{ FLA } & \multicolumn{2}{|l|}{ FLC } \\
\hline & & & & & & Mean $(n=3)$ & SD & Mean $(n=3)$ & SD \\
\hline 1 & 21 & $3.5(0)$ & $30(-1)$ & $105(0)$ & $0.00(-1)$ & 541.4 & 3.8 & 542.4 & 7.9 \\
\hline 2 & 20 & $6.0(+1)$ & $45(0)$ & $200(+1)$ & $0.25(0)$ & 543.4 & 6.5 & 546.4 & 5.6 \\
\hline 3 & 5 & $3.5(0)$ & $45(0)$ & $10(-1)$ & $0.00(-1)$ & 529.2 & 4.0 & 544.0 & 2.0 \\
\hline 4 & 23 & $3.5(0)$ & $30(-1)$ & $105(0)$ & $0.50(+1)$ & 531.6 & 4.4 & 536.6 & 0.8 \\
\hline 5 & 13 & $3.5(0)$ & $30(-1)$ & $10(-1)$ & $0.25(0)$ & 532.0 & 1.8 & 531.0 & 8.1 \\
\hline 6 & 24 & $3.5(0)$ & $60(+1)$ & $105(0)$ & $0.50(+1)$ & 533.2 & 4.7 & 540.0 & 1.7 \\
\hline 7 & 6 & $3.5(0)$ & $45(0)$ & $200(+1)$ & $0.00(-1)$ & 553.6 & 11.7 & 558.0 & 3.4 \\
\hline 8 & 9 & $1.0(-1)$ & $45(0)$ & $105(0)$ & $0.00(-1)$ & 528.0 & 16.4 & 550.2 & 7.1 \\
\hline 9 & 2 & $6.0(+1)$ & $30(-1)$ & $105(0)$ & $0.25(0)$ & 542.4 & 8.2 & 550.6 & 20.2 \\
\hline 10 & 4 & $6.0(+1)$ & $60(+1)$ & $105(0)$ & $0.25(0)$ & 537.6 & 3.0 & 544.2 & 3.8 \\
\hline 11 & 18 & $6.0(+1)$ & $45(0)$ & $10(-1)$ & $0.25(0)$ & 534.0 & 1.4 & 540.4 & 9.6 \\
\hline 12 & 17 & $1.0(-1)$ & $45(0)$ & $10(-1)$ & $0.25(0)$ & 536.8 & 1.4 & 534.4 & 6.9 \\
\hline 13 & 14 & $3.5(0)$ & $60(+1)$ & $10(-1)$ & $0.25(0)$ & 534.8 & 2.7 & 538.8 & 8.8 \\
\hline 14 & 10 & $6.0(+1)$ & $45(0)$ & $105(0)$ & $0.00(-1)$ & 553.2 & 9.6 & 560.6 & 5.6 \\
\hline 15 & 22 & $3.5(0)$ & $60(+1)$ & $105(0)$ & $0.00(-1)$ & 566.8 & 19.9 & 553.2 & 1.7 \\
\hline 16 & 27 & $3.5(0)$ & $45(0)$ & $105(0)$ & $0.25(0)$ & 548.0 & 12.4 & 545.6 & 6.1 \\
\hline 17 & 19 & $1.0(-1)$ & $45(0)$ & $200(+1)$ & $0.25(0)$ & 538.4 & 3.1 & 546.8 & 0.7 \\
\hline 18 & 11 & $1.0(-1)$ & $45(0)$ & $105(0)$ & $0.50(+1)$ & 539.6 & 6.4 & 548.8 & 0.1 \\
\hline 19 & 15 & $3.5(0)$ & $30(-1)$ & $200(+1)$ & $0.25(0)$ & 548.4 & 5.7 & 550.8 & 0.8 \\
\hline 20 & 12 & $6.0(+1)$ & $45(0)$ & $105(0)$ & $0.50(+1)$ & 531.4 & 0.8 & 549.0 & 1.8 \\
\hline 21 & 8 & $3.5(0)$ & $45(0)$ & $200(+1)$ & $0.50(+1)$ & 548.8 & 9.6 & 543.6 & 0.3 \\
\hline 22 & 3 & $1.0(-1)$ & $60(+1)$ & $105(0)$ & $0.25(0)$ & 536.2 & 4.4 & 540.0 & 0.7 \\
\hline 23 & 16 & $3.5(0)$ & $60(+1)$ & $200(+1)$ & $0.25(0)$ & 534.4 & 2.4 & 551.6 & 6.1 \\
\hline 24 & 7 & $3.5(0)$ & $45(0)$ & $10(-1)$ & $0.50(+1)$ & 532.0 & 2.5 & 529.6 & 4.1 \\
\hline 25 & 25 & $3.5(0)$ & $45(0)$ & $105(0)$ & $0.25(0)$ & 533.2 & 1.6 & 531.0 & 9.9 \\
\hline 26 & 1 & $1.0(-1)$ & $30(-1)$ & $105(0)$ & $0.25(0)$ & 531.6 & 0.8 & 540.0 & 7.6 \\
\hline 27 & 26 & $3.5(0)$ & $45(0)$ & $105(0)$ & $0.25(0)$ & 534.2 & 1.6 & 544.2 & 2.1 \\
\hline
\end{tabular}

${ }^{a}$ SD - standard deviation. 


\section{Results and discussion}

\section{Model development for the plasma-reaction systems with the flowing liquid electrodes}

The analysis of the results for the BBD matrix for the plasmareaction system working with the FLA started with their inspection. The variability of the response (the $\lambda_{\max }$ ) between the treatments acquired for 3 independent series of the results was checked. By analyzing a scatter plot overlying the response for all 3 measurement series versus the randomized run order, it was revealed that the variability of the response between the treatments was higher than the variability of the response within the treatments. This could indicate the presence of active parameters in the BBD. Additionally, no correlation between the standard deviation (SD) for the 3 replicated $\lambda_{\max }$ values at given parameters settings and the mean value of the $\lambda_{\max }$ in those conditions was observed; hence, there was no need to stabilize the variance of the response through its transformation. Finally, a scatter plot of the mean value of the $\lambda_{\max }$ versus the randomized run order did not indicate any trend or pattern. Therefore,

Table 2 The ANOVA outputs and the results of the lack-of-fit test for the response surface regression (RSR) models established using the forward selection of terms $(\alpha=0.1)$ for the plasma-reaction system used for the synthesis of the AuNPs with the flowing liquid anode (FLA) or the cathode (FLC) ${ }^{a}$

\begin{tabular}{lccccc}
\hline Source of data & DF & Adjusted SS & Adjusted MS & $F$-Value ${ }^{b}$ & $p$-Value \\
\hline \multicolumn{7}{l}{ The plasma-reaction system with the } & \multicolumn{5}{l}{ flowing liquid anode (FLA) } \\
Model & 6 & 1168.16 & 194.69 & 4.00 & 0.009 \\
Linear & 4 & 747.66 & 186.91 & 3.84 & 0.018 \\
$A$ & 1 & 82.16 & 82.16 & 1.69 & 0.209 \\
$B$ & 1 & 20.28 & 20.28 & 0.42 & 0.526 \\
$C$ & 1 & 387.60 & 387.60 & 7.96 & 0.011 \\
$D$ & 1 & 257.61 & 257.61 & 5.29 & 0.032 \\
2 -Way interactions & 2 & 420.50 & 210.25 & 4.32 & 0.028 \\
$A D$ & 1 & 278.89 & 278.89 & 5.73 & 0.027 \\
$B D$ & 1 & 141.61 & 141.61 & 2.91 & 0.104 \\
Error & 20 & 973.31 & 48.67 & & \\
Lack-of-fit & 18 & 836.48 & 46.47 & 0.68 & 0.744 \\
Pure error & 2 & 136.83 & 68.41 & & \\
Total & 26 & 2141.47 & & &
\end{tabular}

\begin{tabular}{llrrrr}
\multicolumn{6}{l}{ The plasma-reaction system with the flowing liquid cathode (FLC) } \\
Model & 5 & 1125.74 & 225.15 & 10.21 & 0.000 \\
Linear & 3 & 908.22 & 302.74 & 13.73 & 0.000 \\
$A$ & 1 & 80.08 & 80.08 & 3.63 & 0.071 \\
$C$ & 1 & 520.08 & 520.08 & 23.58 & 0.000 \\
$D$ & 1 & 308.05 & 308.05 & 13.97 & 0.001 \\
Square & 2 & 217.52 & 108.76 & 4.93 & 0.018 \\
$A A$ & 1 & 113.68 & 113.68 & 5.15 & 0.034 \\
$D D$ & 1 & 146.82 & 146.82 & 6.66 & 0.017 \\
Error & 21 & 463.20 & 22.06 & & \\
Lack-of-fit & 19 & 333.42 & 17.55 & 0.27 & 0.956 \\
Pure error & 2 & 129.79 & 64.89 & & \\
Total & 26 & 2141.47 & & &
\end{tabular}

${ }^{a} \mathrm{DF}$ - degrees of freedom. SS - sum of squares. MS - mean of squares. $A$ - the flow rate of the flowing liquid electrode. $B$ - the discharge current. $C$ - the Au(III) ions concentration. $D$ - the gelatin concentration. ${ }^{b}$ The value of the $F$-test for comparing the model variance with the residual (error) variance. it was concluded that the plasma-reaction system with the FLA and the AuNP synthesis process in this system were likely not affected by any uncontrolled external parameters.

The response surface, giving the relationship between the $\lambda_{\max }$ and the operating parameters, was approximated with a second-order polynomial function using the least-square regression model and included the linear combinations of the parameters $(A, B, C$ and $D)$, their quadratic combinations $(A A$, $B B, C C$ and $D D)$ and 2-way interactions $(A B, A C, A D, B C, B D, C D)$. The selection of the significant terms in the response surface regression (RSR) model was carried out using a forward selection procedure. The procedure started with no predictors in the model and iteratively added the most significant parameters at each step. It stopped when all parameters not in the model had a $p$-value greater than that specified at the beginning $\alpha$-to-enter value $(\alpha$-to-enter $=0.1)$. The developed RSR full-quadratic model was given as: $\lambda_{\max }=500.3+4.39 \times A+0.483 \times B+$ $0.0598 \times C+99.6 \times D-13.36 \times A D-1.587 \times B D$ (in uncoded units). The analysis of variance (ANOVA) was used to check the model assumptions at a significance level of $\alpha=0.1$. On the basis of the ANOVA output (see details in Table 2), it can be seen that the RSR model is statistically significant (the $F$-value of 4.00 with the $p$-value of 0.009$)$. Additionally, the lack-of-fit test does not identify the lack of fit of the model since the respective $p$-value is at 0.744 . The $R$-squared value for the RSR model was $54.6 \%$ meaning that it explained almost $55 \%$ of the total variance of the $\lambda_{\max }$. The adjusted $R$-squared value was lower (40.9\%), probably due to a couple of insignificant terms. As can be seen from Table 2, the effect of the terms $C, D, A D$ and $B D$, included in the RSR model equation, was significant as the $p$-values for these terms were lower than $\alpha$. The predicted $R$-squared value $(0.4 \%)$ was however close to $0 \%$, pointing out that the developed RSR model for the plasma-reaction system with the FLA could not well predict the response for new observations.

In the case of the plasma-reaction system working with the reversed polarity, where the flowing solution of the AuNP precursor with added gelatin acted as the FLC, the same BBDRSR approach was used. As before, the experiment was repeated 3 times and the resulting series of the responses were considered. The analysis of the results obtained for the BBD matrix (see the input parameters and the output response as the mean values of the $\lambda_{\max }$ along with the SD values in Table 1) also confirmed that the variability between the treatments was dominate. This pointed out that there were active parameters in the BBD. Considering the randomized run order, there was no trend or pattern evident in the data, indicating that the parameters selected for the plasma-reaction system with the FLC were fully controlled. Additionally, this also confirmed that the AuNP synthesis process was not affected by any uncontrolled external parameters. The RSR with the forward selection of the terms $(\alpha$-to-enter $=0.1$ ) was used as before to develop the best model describing the effect of the parameters $A, B, C$ and $D$ on the $\lambda_{\max }$. A full quadratic equation was used to fit the response surface, and the developed RSR model was given as: $\lambda_{\max }=547.36-3.69 \times A+0.0693 \times C-58.6 \times D+0.674 \times A A+$ $76.6 \times D D$ (in uncoded units). The ANOVA evaluation of this 
RSR model is given in Table 2. As can be seen, the model is statistically significant (the $F$-value of 10.21 with the $p$-value < $0.001)$. The relatively high non-significant $p$-value for the lackof-fit test (0.956) also confirmed that the model fits the data. The values of the $R$-squared and the adjusted $R$-squared were high, i.e. $70.8 \%$ and $63.9 \%$, showing the goodness-of-fit of the developed RSR model. Additionally, it seems that the model could well predict the response for new treatments since the predicted $R$-squared value was $53.1 \%$. Considering the $p$-values, the following terms included in the fitted model had a significant effect on the $\lambda_{\max }: A, C, D$ as well as $A A$ and $D D$.

\section{Validation of the developed models}

To validate the RSR models developed for the plasma-reaction system working with the FLA and the FLC, the residuals were analyzed by plotting the normal probability plots (the distribution of the standardized residuals) and the scatter plots of the standardized residuals versus the fitted values. As can be seen from Fig. 2, any unusual observations or deviations from the normal distribution of the residuals can be observed. In the case of the scatter plots of the standardized residuals versus the fitted values, no patterns such as strong curvatures or clusters, which might indicate problems with the regression models, were noted. The points were rather randomly scattered on both sides of 0 . Only single large residuals were identified, i.e. for the run no. 23 in the model of the system with the FLA and run no. 25 in the model of the system with the FLC. This confirmed the correctness of both models and the goodness-of-fit of the experimental data with the RSRs found using the BBD-RSR approach. Considering these observations, it was concluded that there was no reason to reject both models.

On the basis of both RSR model equations, the combination of the input parameters that jointly led to obtaining the response of the lowest $\lambda_{\max }$ value was determined using the respective optimization plots (see Fig. 3). It was established that in the case of the plasma-reaction system working with the FLA, the lowest $\lambda_{\max }$ was obtained for the AuNPs produced when the

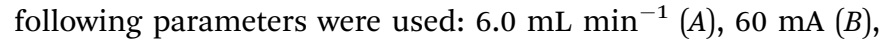
$10.0 \mu \mathrm{g} \mathrm{mL} \mathrm{m}^{-1}(C)$ and $0.50 \%(D)$. The predicted value of the $\lambda_{\max }$ under these conditions was $518.3 \pm 6.5 \mathrm{~nm}$. To validate the RSR model, the plasma-reaction system with the FLA was operated under the established optimal settings. During 3 independently run experiments, the APGD-treated solutions were collected, and the optical properties of the AuNPs synthesized in the above mentioned experimental conditions were determined by UV-Vis absorption spectrophotometry. It was found that the $\lambda_{\max }$ of the LSPR absorption band of the AuNPs was $518.8 \pm 1.1 \mathrm{~nm}$. This value corresponded well with the value predicted by the model and confirmed its reliability; the relative error was only $0.1 \%$.

In a similar way, the optimal settings of the working parameters providing the lowest $\lambda_{\max }$ were derived from the respective optimization plots (see Fig. 3 ) for the plasma-reaction system operated with the FLC. These parameters were $2.7 \mathrm{~mL}$ $\min ^{-1}(A), 10.0 \mu \mathrm{g} \mathrm{mL}{ }^{-1}(C)$ and $0.38 \%(D)$. According to the RSR model, the predicted value of the $\lambda_{\max }$ under these conditions was $531.8 \pm 2.0 \mathrm{~nm}$. During the validation of the model, the plasma-reaction system was operated under the optimal settings, and the APGD-treated solutions containing the synthesized AuNPs were collected and subsequently analyzed by UV-Vis absorption spectrophotometry. It was determined that the $\lambda_{\max }$ of the LSPR absorption band of the produced AuNPs was $526.2 \pm 2.1 \mathrm{~nm}$. This was in a quite good agreement with the predicted value since the relative error was $-1.0 \%$.

\section{The effects of the main parameters on the performance of the plasma-reaction systems}

In the case of the plasma-reaction system sustained between the solid pin cathode and the FLA, the model developed using the BBD-RSR approach showed that the $\lambda_{\max }$ of the LSPR absorption band of the AuNPs produced using this system was affected by the flow rate of the FLA $(A)$, the discharge current $(B)$, the $\mathrm{Au}$ concentration in the solution of the FLA $(C)$ and the gelatin
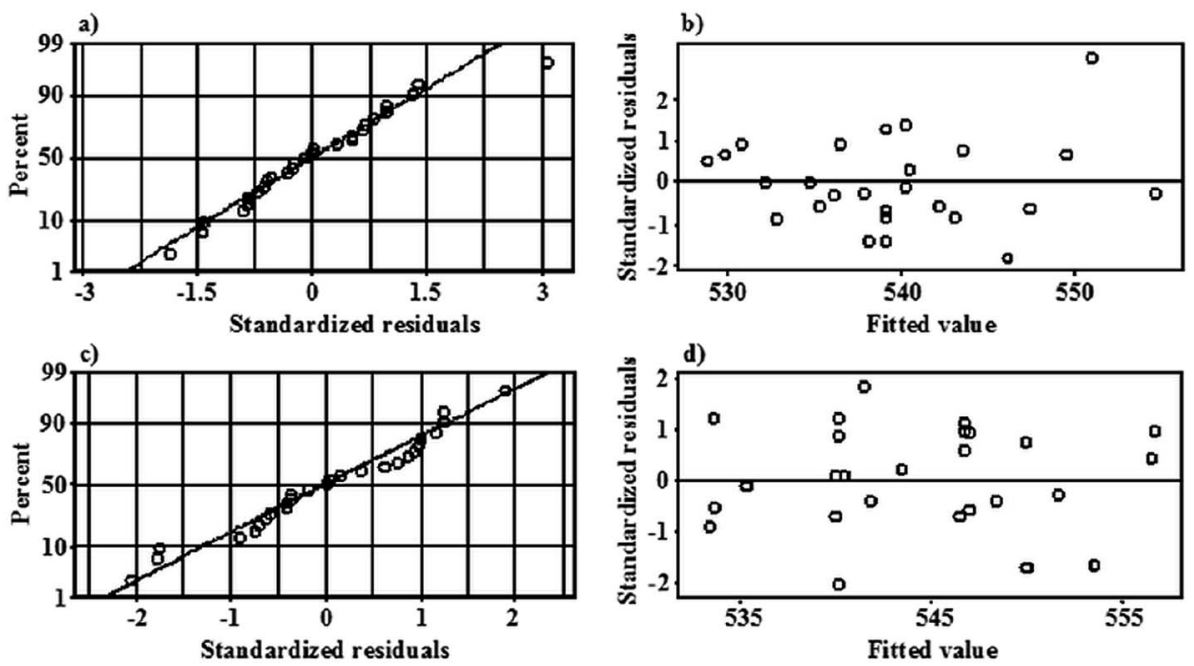

Fig. 2 The distribution of the standardized residuals for the $\lambda_{\max }$ and the standardized residuals versus the fitted values for the RSR models developed for the plasma-reaction system with the FLA ( $a$ and $b$ ) and the FLC (c and d). 


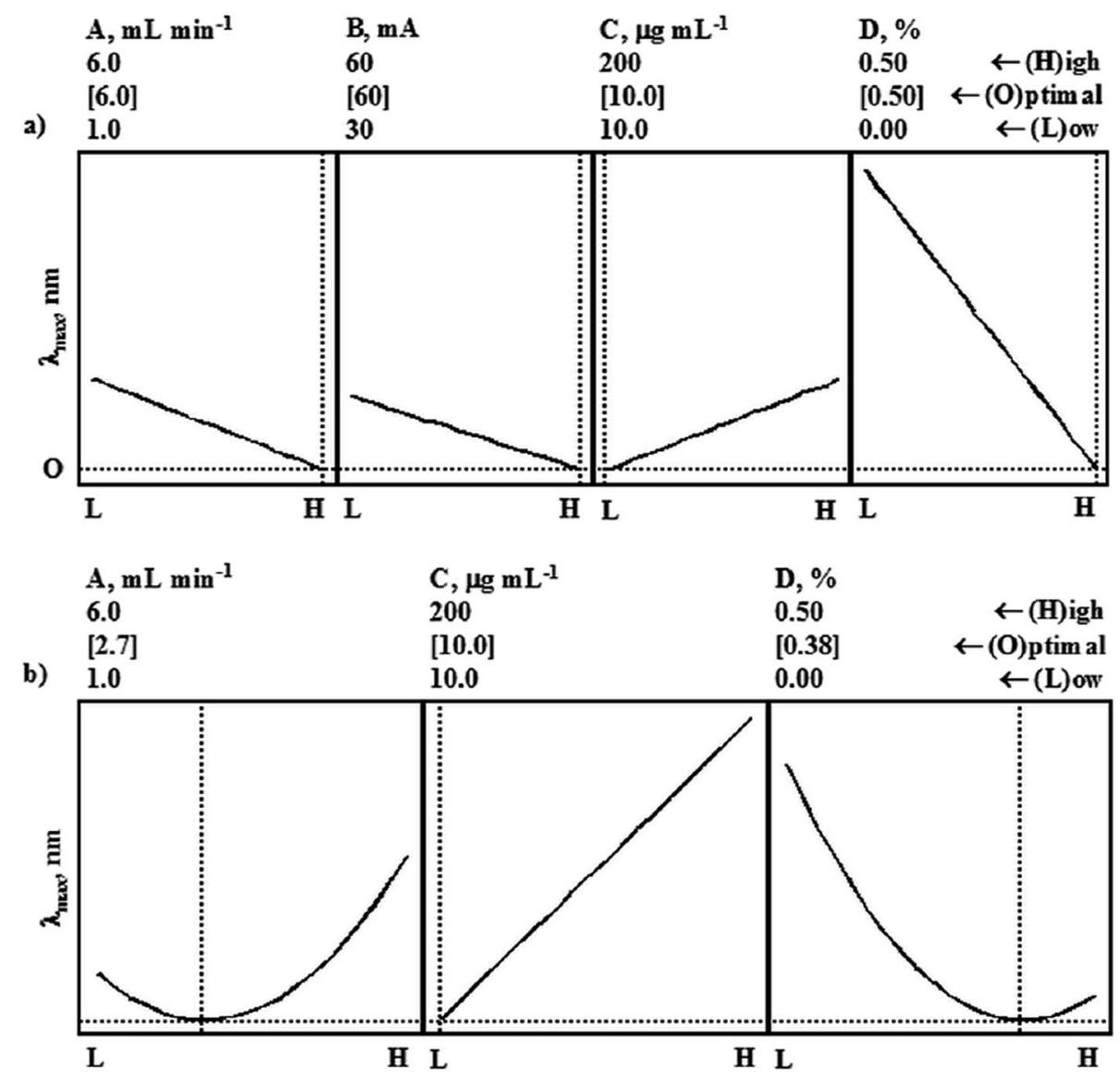

Fig. 3 The effects of the main parameters on the $\lambda_{\max }$ derived from the RSR models developed for the plasma-reaction system with the FLA (a) and the FLC (b).

concentration in the FLA solution $(D)$. A graphical representation of the effects of all 4 main parameters is shown in Fig. 3a with the labeled optimal settings responsible for producing the AuNPs with the smallest particle size that correspond to the lowest value of the $\lambda_{\max }$ in the LSPR absorption band for these nanostructures.

As can be seen, the increase in the flow rate of the FLA (A) and the discharge current $(B)$ leads to a decrease in the $\lambda_{\max }$ as a result of the synthesis of the small sized AuNPs. This behavior could rationally be explained considering the reactions occurring at the plasma-liquid interface under the impact of the electrons on the surface of the FLA solution ${ }^{18-20}$ and the competition existing between the nucleation and growth of the AuNPs in the liquid phase..$^{13}$ It seems coherent that at higher flow rates of the FLA solution (A) the contact time of the APGD phase with the solution containing the $\mathrm{AuCl}_{4}{ }^{-}$ions would be shorter. Hence, the surface-mediated interactions of the resulting nucleation seeds or the growing AuNPs with the partially reduced $\mathrm{AuCl}_{4}{ }^{-}$ions, leading to the formation of larger nanostructures, would be less probable. As a result, very small in size and spherical in shape AuNPs could be produced.

It can also be presumed that the rate of irradiation of the FLA solution surface with the high-energy electrons would be enhanced when the discharge current increased $(B)$. In these conditions, the hydrated electrons could readily be formed, subsequently inducing the formation of the $\mathrm{H}$ radicals. ${ }^{18-20}$ Both the hydrated electrons and the $\mathrm{H}$ radicals could be responsible for the massive reduction of the $\mathrm{AuCl}_{4}{ }^{-}$ions and the development of a great number of nucleation seeds. This could certainly result in the synthesis of the AuNPs with a smaller particle size. Finally, as the concentration of the $\mathrm{Au}(\mathrm{III})$ ions $(C)$ increases (see Fig. 3a), the number of the nucleation seeds would be much higher, thereby significantly reducing the distance between them. Due to the increased probability of interactions between the nucleation seeds and the growing AuNPs as well as the higher amounts of the $\mathrm{AuCl}_{4}{ }^{-}$, larger AuNPs would be synthesized. Similar explanations reference the effects of the discharge current and the concentration of the AuNP precursor, but they were reported for non-flow through systems operated with gaseous ( $\mathrm{Ar}$ or $\mathrm{He}$ ) jets as the cathodes (here, a solid W rod was used and the discharge was fully sustained in the surrounding air) and were lately given by Mariotti et al. ${ }^{9}$ Patel et al., ${ }^{13}$ Wang et al., ${ }^{6}$ and Huang et al. ${ }^{21}$ It was found that the presence of gelatin was required to stabilize the synthesized AuNPs. As can be seen from Fig. 3a, high concentrations of gelatin facilitate the formation of the AuNPs with small particle sizes by preventing their agglomeration and further growth. ${ }^{26}$ The need to use the stabilizers, e.g. fructose ${ }^{21}$ 
or sodium citrate, ${ }^{6}$ in the plasma-mediated synthesis of the AuNPs was reported in other research.

In the case of the plasma-reaction system operated between the solid pin anode and the FLC, the surface of the flowing solutions of the $\mathrm{AuCl}_{4}{ }^{-}$ions was exposed to the positive ions. As compared to the systems with the FLA, it is noted that some of the reactions taking place at the plasma-liquid interface of the system with the FLC and the processes occurring in the liquid phase and accompanying the formation of the AuNPs are different. ${ }^{18,19}$ It is hypothesized that the positive ions bombarding the liquid surface could trigger the dissociative reaction of the $\mathrm{AuCl}_{4}{ }^{-}$ions. ${ }^{20}$ Additionally, the reactive species such as $\mathrm{H}^{+}, \mathrm{OH}^{\cdot}$ and $\mathrm{H}_{2} \mathrm{O}_{2}$ are effectively produced in this case. ${ }^{\mathbf{1 8 , 1 9 , 3 0}}$ According to the developed RSR model, the effect of the discharge current $(B)$ was insignificant on the synthesis process of the AuNPs. Only the flow rate of the FLC solution $(A)$, the AuNP precursor concentration $(C)$ and the gelatin concentration $(D)$ were significant parameters. As can be seen from Fig. 3b, given the optimization plots for this system derived from the RSR regression, at lower flow rates of the FLC solution $(A)$, the plasma-mediated reactions and processes favor the synthesis of the AuNPs with a relatively small particle size as shown by the decreasing $\lambda_{\max }$ values. This could be related to the higher contact time of the solution of the $\mathrm{AuCl}_{4}{ }^{-}$ions with the discharge. ${ }^{30}$ Likely, $\mathrm{H}_{2} \mathrm{O}_{2}$ and the $\mathrm{H}^{+}$ions could efficiently be produced in these conditions, as measured by Jamroz et al., ${ }^{30}$ while the $\mathrm{AuCl}_{4}{ }^{-}$ions could readily dissociate, as presumed by Shirai et al. ${ }^{20}$ All of this together could induce an easier reduction of the $\mathrm{AuCl}_{4}{ }^{-}$ions and the formation of a higher number of nucleation seeds, finally leading to the synthesis of the smallsized and spherical AuNPs. The effect of the decreased $\mathrm{pH}$ on the APGD-treated solutions could also be important and possibly result in etching the AuNPs, making their size smaller and uniform. A similar observation in reference to the effect of the flow rate of the FLC solution on the performance of the AuNP synthesis process was very lately described by Dzimitrowicz et al. $^{27}$ but for a plasma-reaction system operated with an Ar jet and the FLC. The use of higher concentrations of the
$\mathrm{AuCl}_{4}{ }^{-}$ions in the FLC solution was either inconvenient (see Fig. 3b) because it resulted in an increase in the average diameter of the synthesized AuNPs as reflected by the higher $\lambda_{\max }$ values. This was probably related to the enhanced formation of the nucleation seeds in the solution and the higher probability of the interactions of the growing AuNPs with each other and/or with the partly reduced $\mathrm{AuCl}_{4}{ }^{-}$ions as suggested by Patel et $a l .{ }^{13}$ The presence of gelatin in the FLC solution at a relatively high concentration was also required to produce the small-sized AuNPs, likely for the same reasons as in the case of the synthesis process in the plasma-reaction system with the FLA.

\section{Size and shape characterization of the synthesized AuNPs}

To confirm the adequacy and the reliability of both RSR models developed for the plasma-reaction system with the FLA and the FLC on the basis of the $\lambda_{\max }$ of the LSPR absorption band measured by UV-Vis absorption spectrophotometry for the AuNPs present in the APGD-treated solutions, as well as the established effects of the significant parameters, both flowthrough plasma-reaction systems were used to synthesize the AuNPs under optimal and non-optimal, i.e. boundary and mid, experimental conditions (see details in Table 3). Next, the respective APGD-treated solutions were collected, and the resulting AuNPs were further characterized by DLS (no purification of the obtained nanocolloids) as well as SEM and TEM (with a prior purification of the obtained nanocolloids).

As can be seen from Table 3, the results on the particle size of the AuNPs produced in the plasma-reaction system with the FLA, assessed using DLS, coincided very well with the results obtained with UV-Vis absorption spectrophotometry and referred to the location of the $\lambda_{\max }$ of the LSPR absorption band of the synthesized nanostructures. Indeed, the average diameters of the AuNPs produced under the mentioned optimal and non-optimal conditions and measured by DLS revealed changes in the $\lambda_{\max }$ caused by changing the parameter settings from the optimal, to mid and boundary conditions. The linear

Table 3 The compilation of the predicted and measured values of the $\lambda_{\max }$ of the localized surface plasmon resonance (LSPR) absorption band of the AuNPs synthesized in the plasma-reaction system with the flowing liquid anode (FLA) and the cathode (FLC) under the optimal, mid and boundary conditions along with the particle size of the AuNPs obtained in these conditions and measured by dynamic light scattering (DLS)

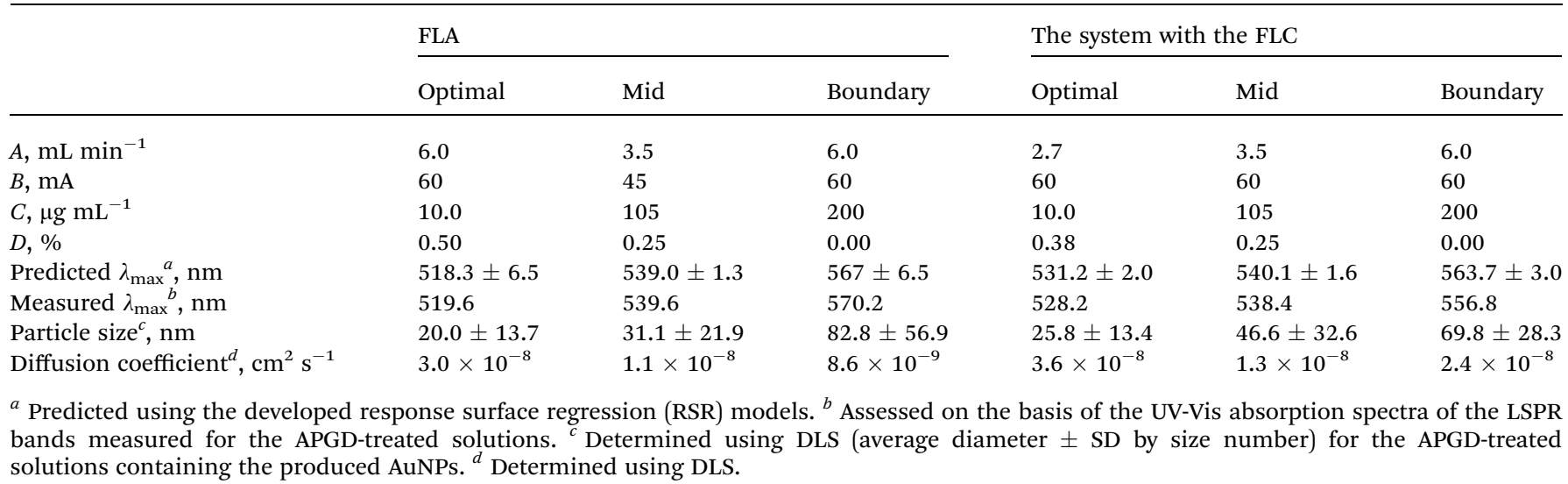



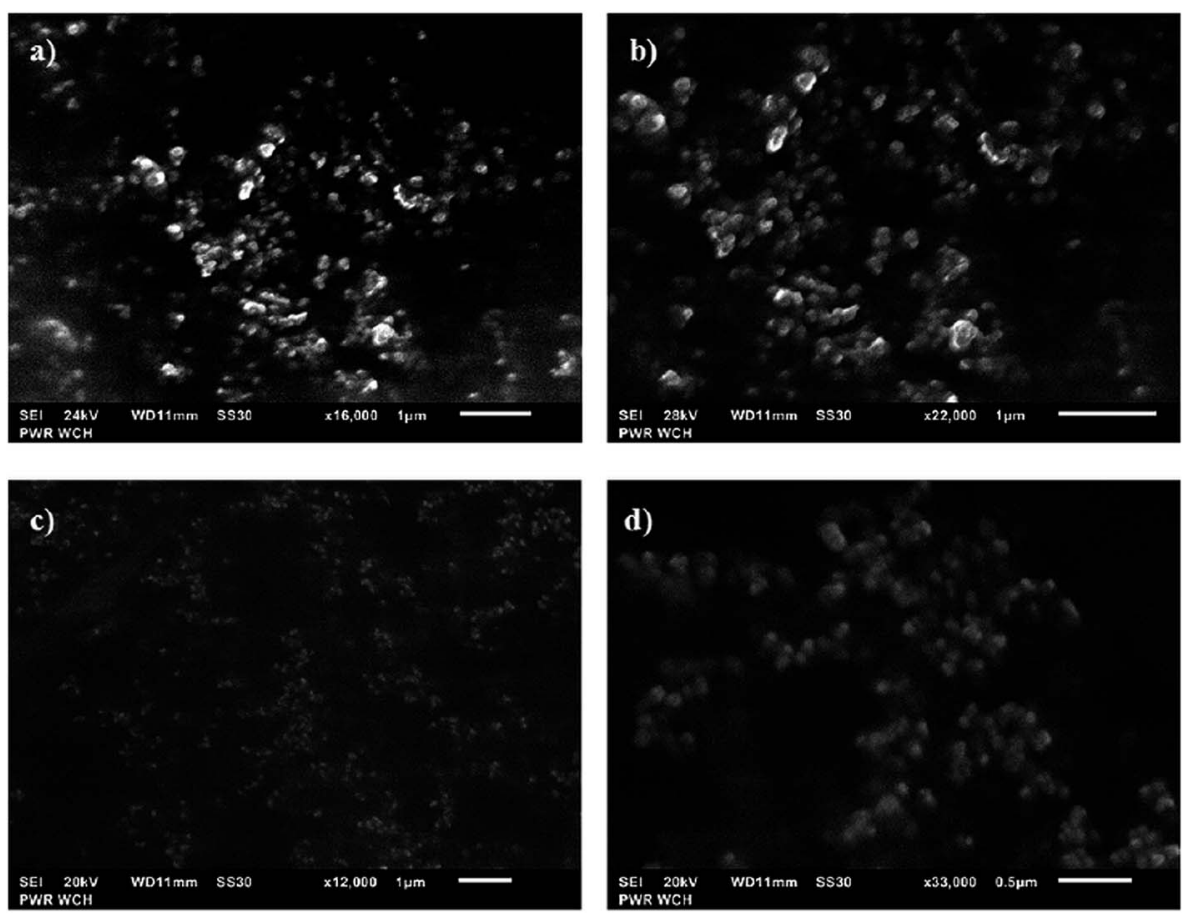

Fig. 4 The representative SEM images of the AuNPs synthesized under the optimal conditions using the plasma-reaction system with the FLA (a and b) and the FLC (c and d).

correlation coefficient $(r)$ between the $\lambda_{\max }$ and the average diameter (as determined by the size number) was 0.973 . For the boundary conditions, the average diameter of the resulting
AuNPs was the highest, while the $\lambda_{\max }$ of the solutions containing the AuNPs produced under these conditions shifted toward the larger wavelengths to the highest degree.
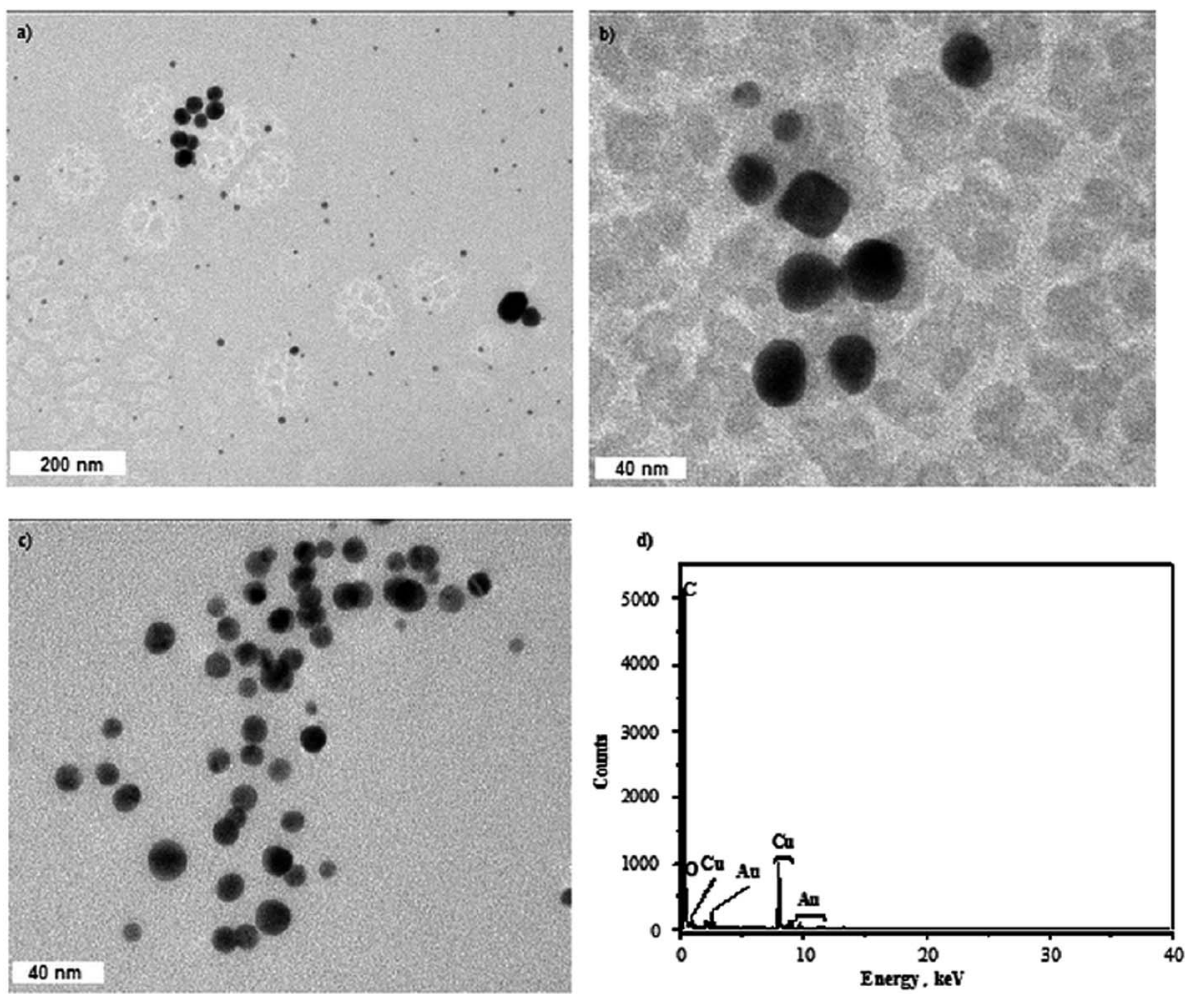

Fig. 5 The representative TEM micrographs of the AuNPs synthesized under the optimal conditions using the plasma-reaction system with the FLA $(a-c)$ along with the EDXMA spectra (d). 

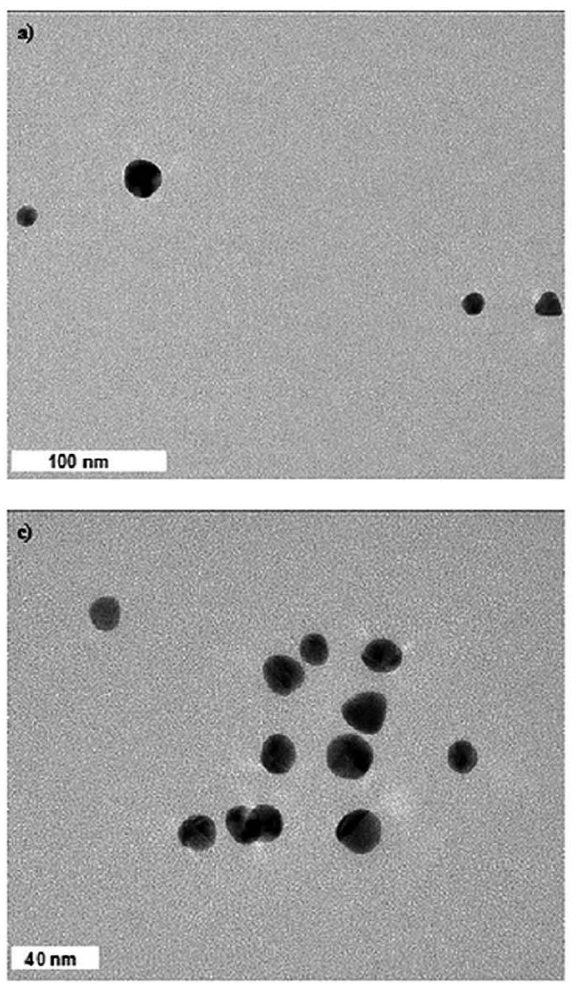
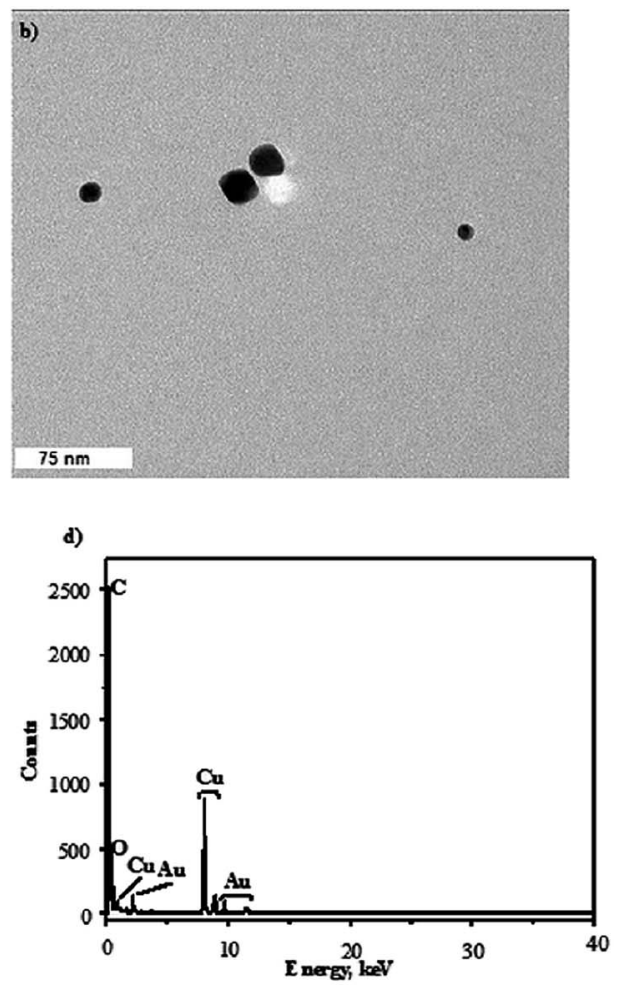

Fig. 6 The representative TEM micrographs of the AuNPs synthesized under the optimal conditions using the plasma-reaction system with the FLC $(a-c)$ along with the EDXMA spectra (d).

An even better coincidence between the measured optical properties and the size of the synthesized AuNPs was observed for the plasma-reaction system with the FLC. In this case, the $r$ between the $\lambda_{\max }$ and the average diameter, as determined by DLS, for the AuNPs produced in the optimal and non-optimal conditions was 0.991 . As compared to the optimal parameters settings, their change to mid and boundary conditions resulted in the production of AuNPs with a bigger particle size that linearly coincided with the red shift of the $\lambda_{\max }$ of the LSPR absorption band.

All findings assessed for the AuNPs synthesized using both plasma-reaction systems under the optimal and non-optimal conditions given in Table 3 were also established to agree with the information retrieved from the SEM and TEM images and related to the particle size of the AuNPs. The representative SEM micrographs of the AuNPs produced in both plasmareaction systems under the optimal conditions, which provide the smallest particle size, are given in Fig. 4a and b (the system with the FLA) and Fig. $4 \mathrm{c}$ and d (the system with the FLC). The representative TEM micrographs of the AuNPs synthesized under the same conditions are given in Fig. 5 (the system with the FLA) and Fig. 6 (the system with the FLC). As can be seen from Fig. 5 and 6, under the optimal parameter settings established on the basis of the developed RSR models, the AuNPs have the smallest diameters, are spherical, uniform (considering relatively narrow particle size distributions) and well dispersed in the medium of the measured nanofluids, with no tendency to agglomerate. The average diameter of the AuNPs synthesized in the plasma-reaction system with the FLA was
$10.9 \pm 7.9 \mathrm{~nm}$ (the calculation was done in the Image software and based on $21 \mathrm{NPs}$ ). In the case of the plasma-reaction system with the FLC, the average diameter of the AuNPs was $13.4 \pm 8.6$ $\mathrm{nm}$ (the calculation was done on the basis of $38 \mathrm{NPs}$ ). These results corresponded to the outcomes provided by the UV-Vis absorption spectrophotometry and DLS measurements. Indeed, by changing the parameter settings to those recognized as boundary or mid (see details in Table 3), the diameters of the resulting AuNPs, retrieved on the basis of the respective TEM micrographs, were enlarged and in shapes other than spherical, i.e. triangular, pentagonal and hexagonal, were present in the measured nanofluids, leading to the broadening of the particle size distribution.

In addition, the long term optical stability was evaluated by measuring over 4 consecutive months the position of the $\lambda_{\max }$ of the representative LSPR absorption band of the spherical AuNPs fabricated under the optimal experimental conditions. It was revealed that the changes in the $\lambda_{\max }$ were lower than $1 \%$. This indicated that the AuNPs synthesized using the plasmamediated synthesis method in the studied plasma-reaction systems were highly stable over time.

\section{Conclusions}

To the best of our knowledge, the present work shows for the first time the production of stable over time AuNPs in a plasmareaction system utilizing the APGD fully operated in the surrounding air between a solid pin electrode, which replaced the gaseous jet, and the FLA or the FLC, depending on the 
polarization of the electrodes. The proposed BDB-RSR approach to the multivariate optimization of the plasma-mediated synthesis of the AuNPs allowed for production of monodispersed and spherical nanostructures in a controllable and predictable way. This fully size-controlled synthesis of the AuNPs appears to be crucial in reference to the future use of the resulting nanocolloids of $\mathrm{Au}$ in relevant technological or medical applications. The smallest AuNPs, as indicated by the UV-Vis absorption spectrophotometry, DLS and TEM, were obtained using the FLA mode, where the solid pin electrode acted as the cathode and the surface of the solution containing the AuNP precursor was irradiated with high-energy electrons. Nevertheless, in the case of both studied plasma-reaction systems, the size of the AuNPs could easily be tuned within the nanometer range by changing the main parameter settings according to the RSR models developed on the basis of the $\lambda_{\max }$ of the LSPR absorption band.

\section{Acknowledgements}

$\mathrm{AD}$ is thankful to the National Science Centre for financial support (UMO-2015/17/N/ST4/03804). PJ and PP also acknowledge the National Science Centre for funding this work (UMO2014/13/B/ST4/05013). In addition, this work was financed by a statutory activity subsidy from the Polish Ministry of Science and Higher Education for the Faculty of Chemistry of Wroclaw University of Technology.

\section{References}

1 M. Bouchard, M. Letourneau, C. Sarra-Bournet, M. LaprisePelletier, S. Turgeon, P. Chevallier, J. Lagueux, G. Laroche and M.-A. Fortin, Langmuir, 2015, 31, 7633.

2 T. Yan, X. Zhong, A. E. Rider, Y. Liu, S. A. Furman and K. Ostrikov, Chem. Commun., 2014, 50, 3144.

3 C. Richmonds, M. Witzke, B. Bartling, S. W. Lee, J. Wainright, C.-C. Liu and R. M. Sankaran, J. Am. Chem. Soc., 2011, 133, 17582.

4 R. Akolkar and R. M. Sankaran, J. Vac. Sci. Technol., A, 2013, 31, 050811.

5 D. Mariotti and R. M. Sankaran, J. Phys. D: Appl. Phys., 2010, 43, 323001.

6 R. Wang, S. Zuo, D. Wu, J. Zhang, W. Zhu, K. H. Becker and J. Fang, Plasma Processes Polym., 2014, 12, 380.

7 D. Mariotti, T. Belmonte, J. Benedikt, T. Velusamy, G. Jain and V. Svrcek, Plasma Processes Polym., 2016, 13, 70.

8 J. McKenna, J. Patel, S. Mitra, N. Soin, V. Svrcek, P. Maguire and D. Mariotti, Eur. Phys. J.: Appl. Phys., 2011, 56, 24020.
9 D. Mariotti, J. Patel, V. Svrcek and P. Maguire, Plasma Processes Polym., 2012, 9, 1074.

10 C. Richmonds and R. M. Sankaran, Appl. Phys. Lett., 2008, 93, 131501.

11 W.-H. Chiang, C. Richmonds and R. M. Sankaran, Plasma Sources Sci. Technol., 2010, 19, 034011.

12 F.-C. Chang, C. Richmonds and R. M. Sankaran, J. Vac. Sci. Technol., A, 2010, 28, L5.

13 J. Patel, L. Nemcova, P. Maguire, W. G. Graham and D. Mariotti, Nanotechnology, 2013, 24, 245604.

14 V. Svrcek, D. Mariotti and M. Kondo, Appl. Phys. Lett., 2010, 97, 161502.

15 D. Mariotti, V. Svrcek, J. W. J. Hamilton, M. Schmidt and M. Kondo, Adv. Funct. Mater., 2012, 22, 954.

16 X. Z. Huang, X. Zhong, Y. Lu, Y. S. Li, A. E. Rider, S. A. Furman and K. Ostrikov, Nanotechnology, 2013, 24, 095604.

17 Y. L. Thong, O. H. Chin, B. H. Ong and N. M. Huang, Jpn. J. Appl. Phys., 2016, 55, 01AE19.

18 F. Tochikubo, N. Shirai and S. Uchida, J. Phys.: Conf. Ser., 2014, 565, 012010.

19 F. Tochikubo, Y. Shimokawa, N. Shirai and S. Uchida, Jpn. J. Appl. Phys., 2014, 53, 126201.

20 N. Shirai, S. Uchida and F. Tochikubo, Jpn. J. Appl. Phys., 2014, 53, 046202.

21 X. Huang, Y. Li and Z. Zhong, Nanoscale Res. Lett., 2014, 9, 572.

22 I. G. Koo, M. S. Lee, J. H. Shim, J. H. Ahn and W. M. Lee, J. Mater. Chem., 2005, 15, 4125.

23 R. Wang, S. Zuo, W. Zhu, J. Zhang and J. Fang, Plasma Processes Polym., 2014, 11, 448.

24 R. Wang, S. Zuo, W. Zhu, S. Wu, W. Nian, J. Zhang and J. Fang, Plasma Processes Polym., 2014, 11, 44.

25 Q. Chen, J. Li and Y. Li, J. Phys. D: Appl. Phys., 2015, 48, 424006.

26 A. Dzimitrowicz, P. Jamroz, K. Greda, P. Nowak, M. Nyk and P. Pohl, J. Nanopart. Res., 2015, 17, 185.

27 A. Dzimitrowicz, T. Lesniewicz, K. Greda, P. Jamroz, M. Nyk and P. Pohl, RSC Adv., 2015, 5, 90534.

28 S. Ghosh, B. Bishop, I. Morrison, R. Akolkar, D. Scherson and R. M. Sankaran, J. Vac. Sci. Technol., A, 2015, 33, 021312.

29 M. A. Bratescu, S.-P. Cho, O. Takai and N. Saito, J. Phys. Chem. C, 2011, 115, 24569.

30 P. Jamroz, K. Greda, P. Pohl and W. Zyrnicki, Plasma Chem. Plasma Process., 2014, 34, 25. 